\title{
INVESTIGATIONS ON THE RECOMBINATION ACTIVITY OF GRAIN BOUNDARIES IN MC SILICON
}

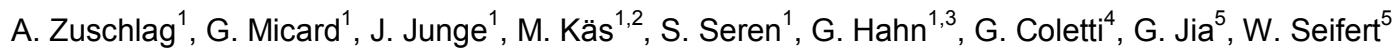 \\ ${ }^{1}$ University of Konstanz, Department of Physics, P.O. Box X916, 78457 Konstanz, Germany \\ 2 now with Berlin Solar $\mathrm{GmbH}$, Magnusstr. 11, 12489 Berlin, Germany \\ ${ }^{3}$ also with Fraunhofer Institute for Solar Energy Systems (ISE), Heidenhofstr. 2, 79110 Freiburg, Germany \\ ${ }^{4}$ ECN-Solar Energy, P.O. Box 1, 1755 ZG Petten, Netherlands \\ ${ }^{5}$ IHP/BTU Joint Lab, BTU Cottbus, Konrad-Wachsmann-Allee 1, 03046 Cottbus, Germany
}

\begin{abstract}
This paper focuses on the influence of the effective intra-grain minority charge carrier diffusion length and surface recombination velocity at grain boundaries on solar cell parameters. Both can be extracted in principle from Light- and Electron Beam Induced Current measurements (LBIC and EBIC). Multicrystalline floatzone (mc FZ) silicon with different grain sizes was processed to solar cells with and without hydrogenation step, followed by LBIC and EBIC characterization. A theoretical model is used which can be applied to measured LBIC or EBIC profiles in order to obtain values for the effective intragrain diffusion length and the recombination velocity at grain boundaries. Efficiencies reached on the processed solar cells (up to $16.0 \%$ ) are the highest reported so far for material with such a small grain size, and the positive effect of hydrogenation can clearly be seen. The obtained results are very useful for other cost effective small grained mc silicon materials.
\end{abstract}

\section{INTRODUCTION}

Besides the impurity level and individual crystal structure the quality of multicrystalline silicon material may be limited by the grain size in terms of a lower effective intra-grain diffusion length for smaller grains compared to larger grains and recombination of minority charge carriers at grain boundaries. To investigate the influence of the grain size on the minority charge carrier diffusion length, mc FZ silicon with different grain sizes is chosen. In addition the beneficial effect of hydrogenation via firing of a hydrogen-rich PECVD $\mathrm{SiN}_{\mathrm{x}}$ layer on the recombination activity of grain boundaries and effective intra-grain diffusion length is of high interest, as the comparably clean grain boundaries of $\mathrm{mc} \mathrm{FZ}$ material can act as a model and the detrimental effect of their recombination activity can be evaluated. On the basis of the experimental LBIC and EBIC data the surface recombination velocity of grain boundaries and intra-grain diffusion length can be extracted for cells processed on neighboring wafers. Therefore we can compare the effect of hydrogenation on the identical grain boundaries if an appropriate model is available. This enables the quantification of the beneficial effect of hydrogenation on the considered parameters and thus allows a quantitative comparison of different hydrogenation techniques and parameters, which could be very helpful for evaluation and determination of the efficiency potential for small grained wafer material.

\section{MULTICRYSTALLINE FZ SILICON}

Due to the production process the crystal quality of block cast mc silicon may suffer from a variety of crystal defects. The effect of the grain size on the crystal quality is normally associated or superposed with the influence of crystal defects such as dislocations and impurities decorating extended crystal defects which complicates the separation of the influence of the grain size on the material quality from experimental data.

Thus for the determination up to which degree the grain size limits solar cell parameters processed from mc material, a very pure material quality is needed to rule out contamination related effects. Such wafers were obtained from mc FZ ingots, grown from a mc seed crystal. The grain size rapidly increases at the beginning of the $F Z$ crystal growth process. Thus wafers with grain sizes in a broad range of a few hundred microns to the $\mathrm{mm}$ scale were available for investigation.

\section{CELL PROCESS AND RESULTS}

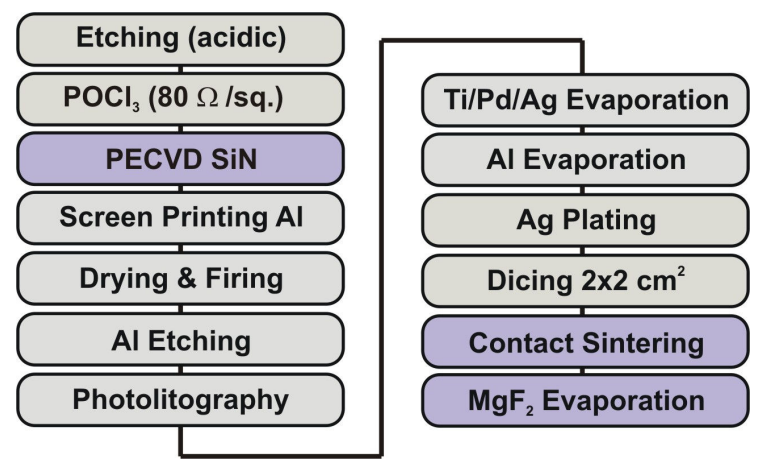

Fig. 1. Photolithography based cell process applied to the mc FZ material. Some processing steps (dark colored) are optional and were not applied to all cells. 
A photolithography based laboratory scale solar cell process was applied to the mc FZ material to demonstrate the material potential and to minimize processing induced defects or limitations.

The cell process shown in Figure 1 consists of an acidic defect etching step, a high efficiency diffused emitter passivated with a thin thermal oxide and a single layer PECVD-SiN antireflective coating (optionally). Further on, the cell process includes a screen printed aluminium back surface field (BSF) and a photolithographically defined front side metallisation realized by the evaporation of $\mathrm{Ti} / \mathrm{Pd} / \mathrm{Ag}$. Rear side metallisation is performed by $\mathrm{Al}$ evaporation followed by Ag plating of the front side metallisation grid. Edge isolation is done by dicing the $5 \times 5 \mathrm{~cm}^{2}$ structure into four $2 \times 2 \mathrm{~cm}^{2}$ cells.

For cells with a hydrogen-rich $\mathrm{SiN}_{\mathrm{x}}$ antireflective coating hydrogenation takes place during the firing step where the $\mathrm{Al} \mathrm{BSF}$ is formed. $\mathrm{A} \mathrm{MgF} \mathrm{M}_{2}$ evaporation of a second layer antireflection coating (DARC) is optional and was only applied to the best $2 \times 2 \mathrm{~cm}^{2}$ cell from each $5 \times 5 \mathrm{~cm}^{2}$ wafer.

In order to investigate the influence of the grain size on the solar cell parameters, mc FZ material with $0.2 \mathrm{~mm}$ and $1 \mathrm{~mm}$ average grain size was processed. For the wafer with the larger grains a neighboring wafer showing an almost identical crystal structure was processed as well without a hydrogen-rich $\mathrm{SiN}_{\mathrm{x}}$ antireflective coating during firing of the Al BSF and without further $\mathrm{MgF}_{2}$ evaporation. The applied cell process results for the larger grained wafer in an enhanced short circuit density most probably as a result of a higher intra-grain effective diffusion length. The achieved efficiencies of $16 \%$ for the larger and $15.5 \%$ for the smaller grained solar cells are the highest reported efficiencies for mc material with such small grains (Table I). Compared to other small grained materials like RGS (grain size around $0.2 \mathrm{~mm}$ ) with a record efficiency of $14.4 \%$ [1], the impurity level of mc FZ silicon is very low which results in higher efficiencies and allows better to separate the influence of the grain size from other crystal defects in further investigations.

\begin{tabular}{|c|c|c|c|c|}
\hline $\begin{array}{c}\text { Grain } \\
\text { size } \\
{[\mathbf{m m}]}\end{array}$ & $\begin{array}{c}\text { FF } \\
{[\%]}\end{array}$ & $\begin{array}{c}\mathbf{V}_{\mathrm{OC}} \\
{[\mathbf{m V}]}\end{array}$ & $\begin{array}{c}\mathbf{J}_{\mathbf{S C}} \\
{\left[\mathbf{m A} / \mathbf{c m}^{2}\right]}\end{array}$ & $\begin{array}{c}\mathbf{n} \\
{[\%]}\end{array}$ \\
\hline \multicolumn{5}{|c|}{ DARC $\left(\mathrm{SiN}_{\mathrm{x}} / \mathrm{MgF}_{2}\right)$} \\
\hline 0.2 & 79.6 & 600 & 32.4 & 15.5 \\
\hline 1 & 79.3 & 599 & 33.7 & 16.0 \\
\hline \multicolumn{5}{|c|}{ no hydrogenation, no ARC } \\
\hline 1 & 76.5 & 551 & 21.4 & 9.0 \\
\hline
\end{tabular}

Table I: IV data of $\mathrm{mc}-\mathrm{FZ}$ solar cells $\left(2 \times 2 \mathrm{~cm}^{2}\right)$ with different grain sizes, antireflective coatings and with optional hydrogenation during processing.

\section{CHARACTERIZATION}

Spatially resolved characterization of the processed mc FZ solar cells was performed in terms of LBIC measurements. In addition EBIC measurements were performed on selected cell areas.

By comparing the LBIC measurements shown in Figure 2 for the cells processed from neighboring wafers with larger grains (B) and (C) it can be found for the cell without the additional hydrogenation via $\mathrm{SiN}_{\mathrm{x}}$ firing $(\mathrm{C})$ that most of the grain boundaries show recombination activity and that the intra-grain IQE and thus diffusion length is lower.
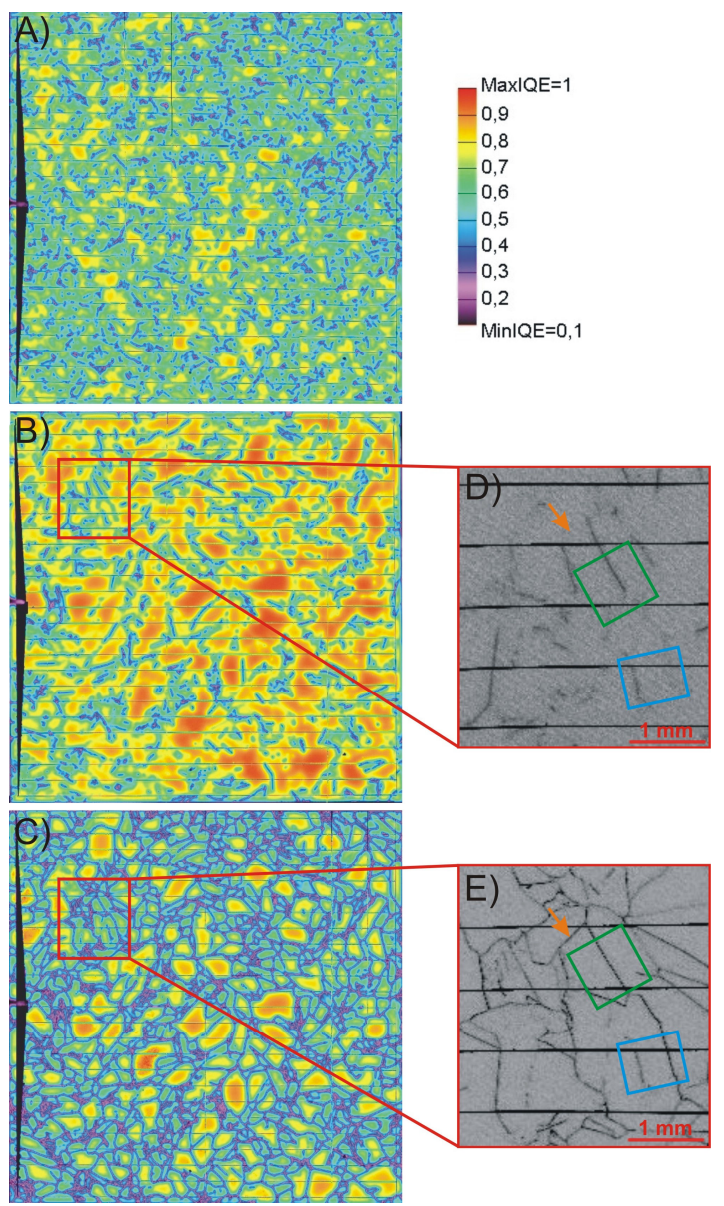

Fig. 2. Internal quantum efficiency (at $980 \mathrm{~nm}$, left, same scaling for all LBIC measurements) and EBIC contrast at $300 \mathrm{~K}$ (right) of mc FZ solar cells with different grain sizes. Grain size of $(A)$ is $0.2 \mathrm{~mm}$, (B) $1 \mathrm{~mm}$ and (C) $1 \mathrm{~mm}$, whereas $C$ is the neighboring wafer to $B$, but without $A R C$ and thus no hydrogenation via $\operatorname{SiN}_{x}$ firing. The marked areas in green and in blue are discussed below. 
For the cells processed with the identical process but from material with different grain sizes (A and $B$ ) it is found that some grain boundaries are still recombination active for both small and large grains. The larger grained cell shows broader areas of a higher IQE. These areas show in addition a higher IQE signal compared to the small grained cell.
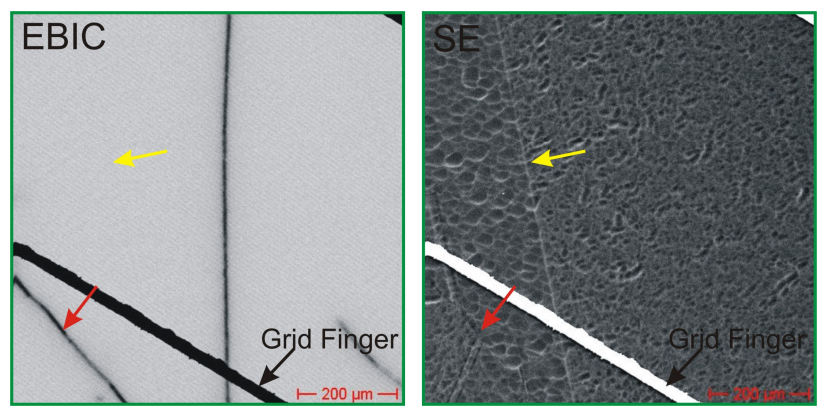

Fig. 3. Detailed EBIC and secondary electron image (green marked area in Figure 2, E) of the larger grained mc-FZ solar cell. The yellow arrow indicates a grain boundary without recombination activity and the red one a change in recombination activity due to a cross over of grain boundaries.

For the areas marked in Figure 2 in addition EBIC measurements were performed. It is found (Figure $2 \mathrm{D}, \mathrm{E}$ ) that particular grain boundaries of the two neighboring wafers show recombination activity. Further on it can be seen, that the recombination activity of the unpassivated large grained cell $(E)$ is sometimes varying along the grain boundary. Besides this it is found that not each grain boundary is necessarily recombination active (yellow arrows in Figure 3). Especially at the crossovers of grain boundaries the recombination activity can change (red arrows in Figure 3).
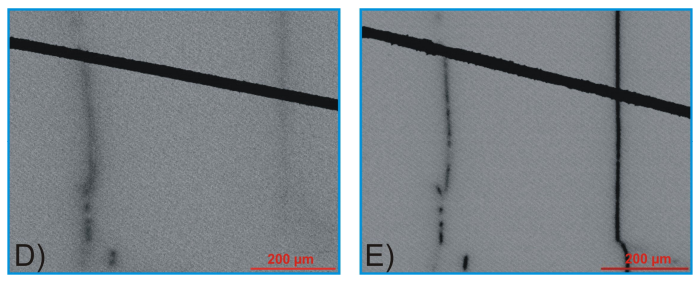

Fig. 4. EBIC measurements at $300 \mathrm{~K}$ of the area marked in blue in Figure 2 ( $D$ and $E$ ) of the cell with and without hydrogenation via SiNx firing.

Both LBIC and EBIC data show for the larger grained solar cells that less grain boundaries are recombination active (Figure 2, D compared to E) depending if hydrogenation was performed during processing. Some grain boundaries seem to be completely passivated while the activity of others is reduced. Figure 4 shows the corresponding grain boundaries of the cells with additional hydrogenation via $\mathrm{SiN}_{\mathrm{x}}$ firing (D) and without (E). The grain boundary at the right side of Figure $4(\mathrm{E})$ is more recombination active compared to the grain boundary on the left in Figure $4(\mathrm{E})$, but the cell with the additional hydrogenation via $\mathrm{SiN}_{\mathrm{x}}$ firing shows a reversed behavior (Figure 4 (D)). The recombination activity of the grain boundary on the right vanishes nearly whereas the influence of the passivation is very low for the left grain boundary where a more diffuse contrast can be seen after hydrogenation. This could be a hint for "bleeding" of impurities that have been located close to the grain boundary.

A low temperature EBIC measurement of the region shown in Figure 2 (D) reveals more details concerning recombination active defects in the material. In particular, it is found, that dislocations with substantially varying area density are present as well (Figure 5).

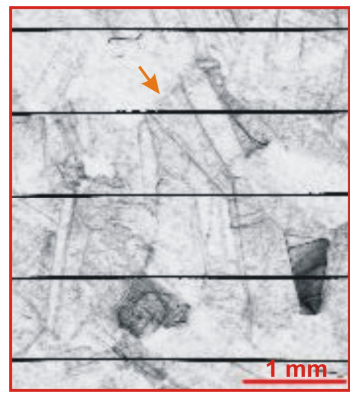

Fig. 5. Low temperature ( $77 \mathrm{~K})$ EBIC measurements of area (D) shown in Figure 2.

Compared to the EBIC data of the cell without hydrogenation via $\mathrm{SiN}_{\mathrm{x}}$ firing, some grain boundaries show even at the low temperature measurement no recombination activity as a result of the hydrogenation (orange arrows in Figure 2 (D), (E) and Figure 5).

\section{MODELING}

From the experimental LBIC maps, line-scans were extracted perpendicularly to the grain boundaries and normalized to the level obtained infinitely far from these grain boundaries (plateau level). These normalized linescans, the so called contrast profiles, allow the determination of the surface recombination velocity at the grain boundary as well as the diffusion length of the minority charge carriers in each grain. The evaluation of these parameters is carried out making use of a theoretical model based on the solution of the carrier flow continuity equations [2] with suitable boundaries conditions. We used here the direct fitting procedure approach which allows better accuracy of the extracted values since none of the information contained in the experimental contrast data is discarded [3].

We successfully implemented this model for LBIC profiles considering the Gaussian power density profile of our laser. The main difficulty of this approach lies in the choice of the grain boundary and the position of the linescan which is not trivial because of possible non symmetry of the LBIC profiles [4]. These asymmetries can in principle result from different intra-grain diffusion lengths 
of the neighboring grains affecting each other, the proximity of other grain boundaries or a grain boundary not lying perpendicular to the surface. For this reason, we chose straight grain boundaries between the largest grains, which can be found on the hydrogenated and also on the neighboring wafer without hydrogenation.

In the case of the large grained wafer, we studied two grain boundaries (GB1 and GB2) which were differently affected by hydrogenation via $\mathrm{SiN}_{\mathrm{x}}$ firing (fitted parameters given in Table II).

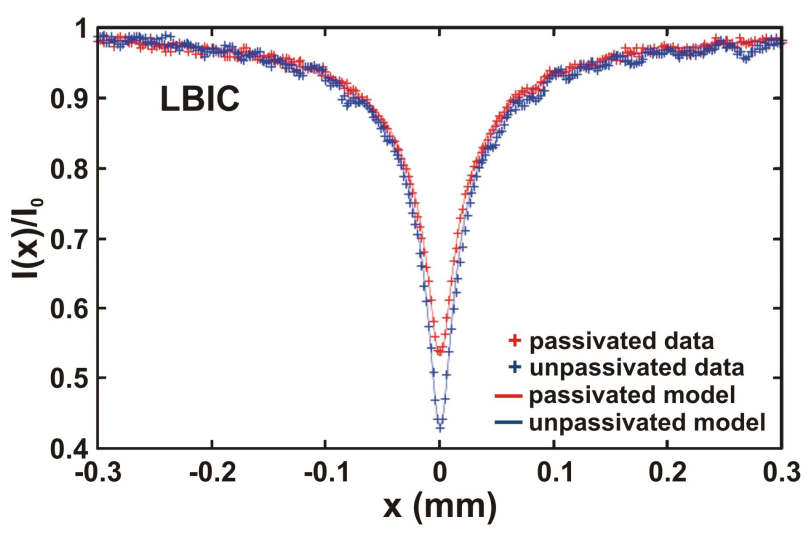

Fig. 6. Contrast profiles of the LBIC measurements and fits for the large grained wafer with and without hydrogenation.

In the case of GB1 (Figure 6) the surface recombination velocity decreases due to hydrogenation while the estimated diffusion length remains constant at a very high value $(1 \mathrm{~mm})$, which is consistent with the high quality of the mc FZ material. For this grain boundary, the difference in diffusion length between the hydrogenated and non hydrogenated case cannot be accurately estimated due to the relative insensitivity to changes in diffusion length when the collection efficiency is high [3] and, probably, a not so large difference.

\begin{tabular}{|c|c|c|c|}
\cline { 3 - 4 } \multicolumn{2}{c|}{} & GB1 & GB2 \\
\hline \multirow{2}{*}{ passivated } & $\mathrm{L}_{\text {Diff }}[\mathrm{mm}]$ & 1 & 1.1 \\
\cline { 2 - 4 } & $\mathrm{SRV}[\mathrm{cm} / \mathrm{s}]$ & $6.8^{*} 10^{4}$ & $4.5^{\star} 10^{4}$ \\
\hline \multirow{2}{*}{ unpassivated } & $\mathrm{L}_{\text {Diff }}[\mathrm{mm}]$ & 1 & 0.44 \\
\cline { 2 - 4 } & $\mathrm{SRV}[\mathrm{cm} / \mathrm{s}]$ & $1.7^{*} 10^{5}$ & $2.4^{*} 10^{5}$ \\
\hline
\end{tabular}

Table II: Calculated values of the diffusion length and the surface recombination velocity extracted from LBIC measurements on grain boundaries 1 and 2 .

Low temperature EBIC measurements show that the dislocation density in the two neighboring grains is very low, which could explain the high diffusion length in the non hydrogenated case and why it is not improving after hydrogenation.

In the case of GB2 the surface recombination velocity decreases even more than for GB1 while the diffusion length increases by more than $100 \%$. This could be explained by considering that the density of defects is higher in the GB2 than in the GB1 area. Indeed, lower diffusion length and higher surface recombination velocity, respectively, indicate a higher density of defects around and at this grain boundary. The difference in density of defects between the GB1 and GB2 area could be attributed to defects that are passivated by hydrogenation. As comparable diffusion lengths can be found for both areas after hydrogenation, we can state that the density of non passivated defects is qualitatively the same for both areas after hydrogenation, limiting the intra-grain diffusion length to the same value of around $1 \mathrm{~mm}$.

\section{SUMMARY}

Solar cells were processed from mc FZ silicon of different grain sizes according to a laboratory-type photolithography based cell process, leading to the efficiencies of up to $16.0 \%$. The cells were investigated in a spatially resolved manner by LBIC and EBIC measurements. Corresponding crystal structures of neighboring wafers were studied in detail. It was found that the grain size as well as an applied hydrogenation during the cell process has strong influence on intra-grain effective diffusion length and recombination activity of grain boundaries. The performance of the mc FZ solar cells under investigation seems to be limited by the size of the grains. Other low cost materials with comparable grain size like RGS (Ribbon Growth on Substrate) silicon are currently limited to lower efficiency values. This can be explained by other intra-grain defects (e.g. impurities), and therefore grain size in general is not the most prominent limiting factor for efficiency in these materials. Applying a fitting procedure to the LBIC grain boundaries contrast profile gave an estimation of diffusion lengths and of surface recombination activity for hydrogenated and non hydrogenated solar cells, which allowed a more quantitative evaluation of the efficiency of the hydrogenation step.

\section{OUTLOOK}

The gathered data as partially presented within this paper will be investigated further in order to find correlations between grain size, crystal orientation or other crystal related characteristics and the resulting solar cell characteristics. Values for the effective intra-grain diffusion length and the surface recombination velocity at grain boundaries are presently obtained from the measurements by a model assuming the same diffusion length for both neighboring grains. However, the model is currently expanded to a more general situation allowing different diffusion lengths and/or the influence of another nearby grain boundary as well. Some of the assumptions used for the fitting procedure and their effect on fit results (e.g. influence of cell thickness) still have to be investigated further. 


\section{ACKNOWLEDGEMENTS}

Part of this work was funded by the EC in the CrystalClear (SES6-CT-2003-502583) and the German $\mathrm{BMU}$ in the SolarFocus $(0327650 \mathrm{H}, 0327650 \mathrm{~A})$ project. The content of this publication is the responsibility of the authors.

\section{REFERENCES}

[1] S. Seren, M. Kaes, G. Hahn, A. Gutjahr, A.R. Burgers, A. Schönecker, "Efficiency potential of RGS silicon from current R\&D production", Proc. $22^{\text {nd }} E U$ PVSEC, Milan 2007, 854

[2] C. Donolato, "Theory of beam induced current characterization of grain boundaries in polycrystalline silicon", J. Appl. Phys. 54, 1314, 1983

[3] R. Corkish, T. Puzzer, A.B. Sproul, K.L. Luke, "Quantitative interpretation of electron-beam-induced current grain boundary contrast profiles with application to silicon“, J. Appl. Phys. 84 (10), 5473, 1998

[4] O. v. Roos, K.L. King, "Analysis of the electronbeam-induced current of a polycrystalline $p-n$ junction when the diffusion lengths of the material on either side of a grain boundary differ", J. Appl. Phys. 55, 4275, 1984 